MATEC Web of Conferences 11,01005 (2014)

DOI: $10.1051 /$ matecconf $/ 20141101005$

(C) Owned by the authors, published by EDP Sciences, 2014

\title{
Elaboration d'une Pouzzolane Artificielle à Base de la Zéolithe X Synthétisée à partir de Kaolin Naturel
}

\author{
Anissa D. Benredouane ${ }^{1}$, Larbi Kacimi ${ }^{1 *}$, Olga Rodriguez Largo ${ }^{2}$ and Aurora Lopez Delargo ${ }^{2}$ \\ ${ }^{1}$ Laboratoire des Eco-Matériaux Fonctionnels et Nanostructurés, Université des Sciences et de la Technologie d'Oran, \\ B.P. 1505 El- M’Naouer, U.S.T.O., Oran, Algérie. \\ ${ }^{2}$ Centre National des Recherches Métallurgique (CENIM), Conseil Supérieur des Recherches Scientifiques (CSIC), \\ Madrid, Espagne.
}

\begin{abstract}
Résumé. Cette étude est une contribution à l'élaboration d'une nouvelle pouzzolane artificielle à base de zéolithe $\mathrm{X}$ synthétisée à partir de kaolin naturelle. Le choix de cette zéolithe revient à son pouvoir d'adsorption qui lui permet de réagir facilement avec la portlandite dans la pâte de ciment. La synthèse de cette zéolithe, sa caractérisation et les tests de sa pouzolanicité, ainsi que l'élaboration de ciment pouzzolanique à base de cette zéolithe et l'étude de ses caractéristiques physiques et mécaniques constituent l'objet de ce modeste travail. L'activité pouzzolanique de cette zéolithe a été déterminée par le test de chaux saturée, par diffraction des rayons X et par analyse thermique (ATG-DSC). Des essais physiques (temps de prise) et mécaniques (résistances à la compression et à la flexion) ont été effectués sur des pâtes et mortiers de ciment renfermant 0 à $20 \%$ de zéolithe synthétisée. Les résultats obtenus sont très satisfaisants et permettent de conclure que la zéolithe $\mathrm{X}$ synthétisée est fortement pouzzolanique et peut être utilisée pour élaborer un éco-ciment tout en réduisant les émissions de $\mathrm{CO}_{2}$ résultant de la fabrication du clinker largement substitué par cette zéolithe dans le ciment Portland.
\end{abstract}

\section{Introduction}

La grande consommation de l'énergie (thermique et électrique) et les fortes émissions de $\mathrm{CO}_{2}$ (gaz à effet de serre) constituent les principaux défis pour l'industrie de ciment. Ces deux problèmes résultent de la fabrication du clinker du ciment, dont sa cuisson à haute température $\left(1450-1500^{\circ} \mathrm{C}\right)$ nécessite une grande quantité de combustible, et de l'utilisation d'un grand pourcentage de calcaire $(75 \%$ du mélange $\mathrm{cru})$ riche en carbonate de calcium

Une des alternatives, adoptées par les technologues et les chercheurs dans le domaine du ciment, pour remédier à ces problèmes, est de substituer une partie de clinker par des ajouts dans le ciment Portland pour fabriquer ce que l'on appelle «ciment composé ». Ces ajouts peuvent être inertes ou actifs; dans le deuxième cas (ajout actif) le ciment est appelé pouzzolanique et cet ajout est désigné par le terme « pouzzolane » [1-3].

La pouzzolane est un minérale riche en silice $\left(\mathrm{SiO}_{2}\right)$ et/ou alumine $\left(\mathrm{Al}_{2} \mathrm{O}_{3}\right)$ réactifs. La réactivité de ces deux oxydes leur permet de réagir facilement avec la portlandite $\left[\mathrm{Ca}(\mathrm{OH})_{2}\right]$ résultant de l'hydratation des minéraux du clinker, $3 \mathrm{CaO} . \mathrm{SiO}_{2} \quad\left(\mathrm{C}_{3} \mathrm{~S}\right)$ et $2 \mathrm{CaO} \cdot \mathrm{SiO}_{2}$ $\left(\mathrm{C}_{2} \mathrm{~S}\right)$, en formant plus de gel de C-S-H responsable du durcissement et du développement de la résistance mécanique de la pâte de ciment.

Les pouzzolanes peuvent être naturelles ou artificielles. La pouzzolane naturelle est une roche volcanique renfermant une certaine quantité de silice présentant une certaine réactivité. Par contre, les pouzzolanes artificielles sont des résidus de certaines industries, telles que les cendres volantes des centrales thermiques et la fumée de silice, qui renferment une quantité importante de slice et d'alumine réactives.

Néanmoins, ces pouzzolanes n'ont pas une réactivité élevée qui leur permet de substituer plus de $10 \%$ de clinker sans affecter les performances mécaniques de la pâte de ciment. Ces dernières années les chercheurs se sont orientés vers l'élaboration de nouvelles pouzzolanes de haute réactivité pour substituer le maximum de clinker dans un ciment considéré écologique (éco-ciment) tout en préservant les propriétés mécaniques de la pâte de ciment. Cette élaboration peut être réalisée par plusieurs méthodes de traitement de matériaux d'aluminosilicates : mécanique (broyage poussé), thermique (calcination), chimique (attaque alcaline) et hydrothermale (chauffage sous pression d'une solution liquide du matériau) [4,5].

Cette étude est une contribution à l'élaboration d'une nouvelle pouzzolane à base de zéolithe $\mathrm{X}$ synthétisée à partir de kaolin naturelle. Le choix de cette zéolithe 
revient à son pouvoir d'adsorption qui lui permet de réagir facilement avec la portlandite dans la pâte de ciment. Des caractérisations de la zéolithe synthétisée et des tests de sa pouzolanicité, par DRX, ATG et essai de chaux saturée, ainsi que des essais physiques et mécaniques du ciment pouzzolanique élaboré à base de cette zéolithe ont été réalisés.

\section{Expérimentation}

La composition chimique du kaolin naturel utilisé, déterminée par fluorescence $\mathrm{X}$, à la cimenterie de Sig (CiBA) du Groupe Lafarge est donnée dans le Tableau 1. La composition minéralogique de ce kaolin, déterminée par Diffraction des Rayons X (DRX), est donnée dans la figure 1 .

Tableau 1. Composition chimique ( $\%$ massique) du kaolin naturel utilisé

\begin{tabular}{|l|l|l|l|l|l|l|l|l|l|l|}
\hline 颔 & $\mathrm{Si}_{2}$ & $\mathrm{Al}_{2} 0_{3}$ & $\mathrm{Fe}_{2} 0_{3}$ & $\mathrm{Ca}_{\mathrm{a}} 0$ & $\mathrm{Mg}_{\mathrm{g}} 0$ & $\mathrm{~S}_{3}$ & $\mathrm{Na}_{2} 0$ & $\mathrm{~K}_{2} 0$ & $\mathrm{P}_{2} 0_{5}$ & P.F. \\
\hline 消 & 65,71 & 21,56 & $\mathbf{0 , 9 4}$ & 0,21 & $\mathbf{0 , 5 9}$ & 0,05 & $\mathbf{2 , 6 2}$ & 2,24 & 0,06 & $\mathbf{6 , 5}$ \\
\hline
\end{tabular}

Selon la composition chimique (Tableau 1), le kaolin utilisé est riche en silice et alumine, avec un rapport $\mathrm{SiO}_{2} / \mathrm{Al}_{2} \mathrm{O}_{3}=3$. D'autre impureté peuvent exister comme $\mathrm{Na}_{2} \mathrm{O}$ et $\mathrm{K}_{2} \mathrm{O}$. Ce kaolin renferme en plus de la kaolinite, du mika et du quartz (figure 1).

Le ciment utilisé est de type CEM I-CRS-42,5N de la cimenterie de Sig de la compagnie Lafarge. Il est composé de $95,5 \%$ de clinker et $4,5 \%$ de gypse. Du fait qu'il est composé uniquement de clinker avec un pourcentage faible de $\mathrm{SO}_{3}$, ce ciment est utilisé dans cette étude pour tester la pouzzolanicité de la zéolithe en élaborant un ciment zéolithique et déterminant ses proriétés physiques (prise) et mécaniques (resistance à la compression).

La composition chimique du ciment utilisé, déterminée par Fluorescence $\mathrm{X}$, est donnée dans le Tableau 2.

Tableau 2. Composition chimique (\% massique) du ciment CEM I $42,5 \mathrm{~N}$

\begin{tabular}{|l|l|l|l|l|l|l|l|l|l|}
\hline Oxyde & $\mathrm{Ca}_{2}$ & $\mathrm{Si}_{2}$ & $\mathrm{Al}_{2} \mathrm{O}_{3}$ & $\mathrm{Fe}_{2} \mathrm{O}_{3}$ & $\mathrm{SO}_{3}$ & $\mathrm{~K}_{2} \mathrm{O}$ & $\mathrm{Na}_{2} 0$ & $\mathrm{Mg}_{0} 0$ & $\mathbf{C ~ I}$ \\
\hline Pourcentage & 63,34 & 21,16 & $\mathbf{3 , 7 7}$ & $\mathbf{5 , 0 4}$ & 2,42 & 0,49 & $\mathbf{0 , 1 6}$ & $\mathbf{2}, 13$ & $\mathbf{0 , 0 2 5}$ \\
\hline
\end{tabular}

La composition minéralogique, déterminée par la méthode de Bogue, montre que le clinker de ce ciment est composé de : $57,5 \% \quad \mathrm{C}_{3} \mathrm{~S} ; 20,2 \% \quad \mathrm{C}_{2} \mathrm{~S} ; 2,5 \% \quad \mathrm{C}_{3} \mathrm{~A}$; $15,3 \% \mathrm{C}_{4} \mathrm{AF} ; 0,8 \% \mathrm{CaO}$ libre.

La diffraction des rayons $\mathrm{X}$ et l'analyse thermogravimétrique ont été utilisées pour caractériser le matériau utilisé (kaolin naturel) et la zéolithe $\mathrm{X}$ synthétisée, ainsi que pour tester l'activité pouzzolanique de cette zéolithe en préparant des pâtes de mélange zéolithe-chaux hydraulique. La pouzzolanicité de la zéolithe synthétisée a été aussi évaluée par le test de chaux saturée et par l'étude de la prise et la résistance à la compression à 2,7 et 28 jours de durcissement du ciment composé de 0,10 et $20 \%$ de cette zéolithe.

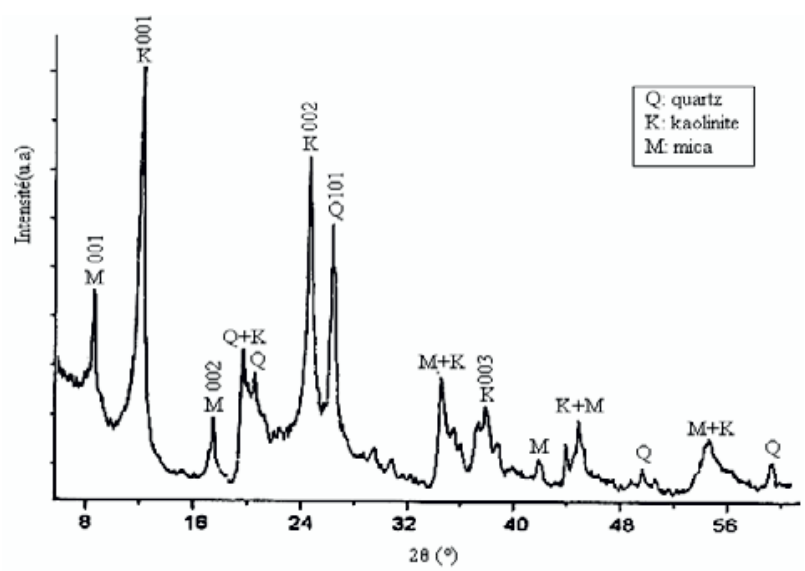

Fig. 1. Diffractogramme des Rayons X du kaolin naturel utilisé

\section{Résultats et Discussions}

\subsection{Synthèse de la zéolithe $X$ à partir du kaolin naturel}

Malgré la teneur modérée de kaolinite dans le kaolin naturel et la présence d'impuretés, la zéolithe $\mathrm{X}$ a été formée avec des intensités fortes des pics caractéristiques de ce matériau (figure 2). Le quartz est rencontré dans cette zéolithe avec d'autres impuretés en très faibles intensités. Une phase amorphe est également formée dans ce produit, ce qui est présentée par une bosse à $2 \theta / 20-50^{\circ}$.

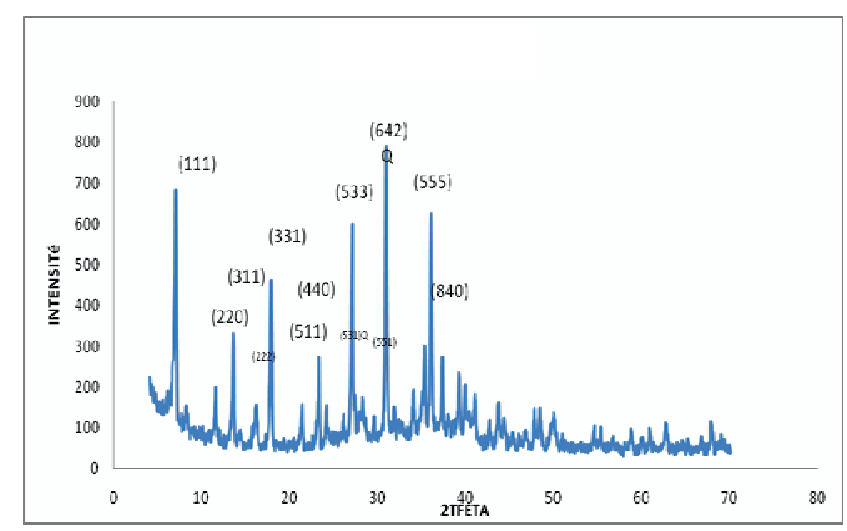

Fig. 2. Diffractogramme RX de la zéolithe $\mathrm{X}$ synthétisée à partir de kaolin naturel

\subsection{Etude de la pouzzolanicité de la zéolithe $X$ par le test de chaux saturée}

L'activité pouzzonalique de la zéolithe $\mathrm{X}$ synthétisée est déterminée par le pourcentage de $\mathrm{CaO}$ consommé en utilisant le test de chaux saturée. La figure 3 donne les pourcentages de $\mathrm{CaO}$ consommé par cette zéolithe. 


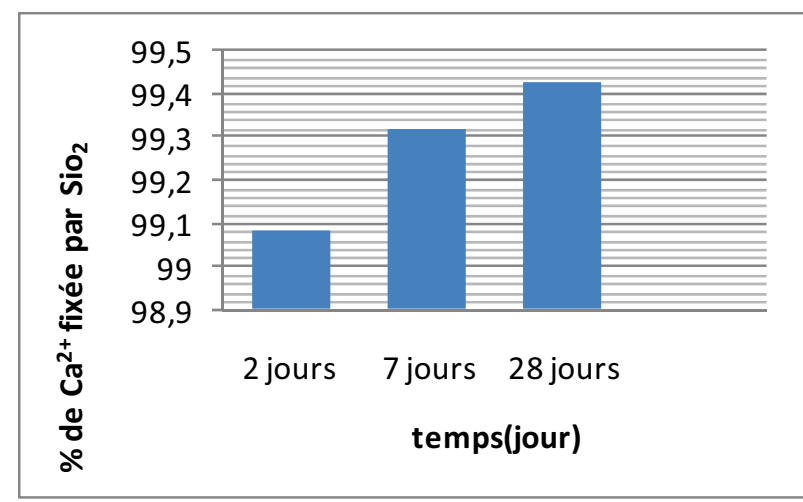

Fig. 3. Variation de l'activité pouzzolanique $(\% \mathrm{CaO}$ moyen consommé) de la zéolithe $\mathrm{X}$ synthétisée en fonction du temps

Selon les résultats obtenus, le pourcentage de la chaux consommée par la zéolithe $\mathrm{X}$ est très élevé et depasse $99 \%$ à toutes les échéances avec une augmentaion dans le temps pour attendre une valeur de $99,5 \%$ à 28 jours. Ceci montre que cette zéolithe est caratéisée par une activité pouzzolaniue très importante, ce qui permet de son utilisation avec un grand pourcentage dans le ciment.

\subsection{Etude de la pouzzolanicité de la zéolithe synthétisée par DSC-ATG}

Pour confirmer l'activité pouzzolanique de la zéolithe $\mathrm{X}$ synthétisée, l'analyse thermique (DSC-ATG) a été utilisée pour déterminer le taux de la chaux consommée par la zéolithe après 28 jours d'hydrataion. Cette technique est appliquée pour des pâtes de mélanges (Zéolithe X- Chaux hydratée) avec $1 / 3$ de chaux $\left[\mathrm{Ca}(\mathrm{OH})_{2}\right]$ et $2 / 3$ de zéolithe $X$ synthetisée après 28 jours de durcissement. Le taux de chaux consommée est déduit de la masse de portlandite résiduelle montrée par la perte de masse enregistrée sur le diagramme DSC-ATG. Les résultats obtenus sont illustrés dans la figure 4.

Une grande perte de masse (12\%) est remarquée entre 100 et $200^{\circ} \mathrm{C}$ en $\mathrm{ATG}$ avec un grand pic endothermique en DSC. Ceci montre la déshydrataion des C-S-H/C-A-H formés par la réaction entre la portlandite d'une part, et la silice et l'alumine de la zéolithe d'autre part. Cette grande perte de masse est accompagnée par une faible variation de la masse $(0,18 \%)$ entre 400 et $500^{\circ} \mathrm{C}$ et un faible pic endotermique en DSC qui est le domaine de déshydrataion de la portlandite ce qui témoigne la faible présence de la chaux hydratée $\left[\mathrm{Ca}(\mathrm{OH})_{2}\right]$. Ces constatations montrent qu'une grande quantité de la chaux hydratée s'est transformée en gels de C-S-H/C-A$\mathrm{H}$ par la réaction pouzzolanique entre la chaux hydraulique et la silice ou l'alumine de la zéolithe synthétisée dans la pâte de mélange, ce qui confirme la pouzzolanicité de cette zéolithe.

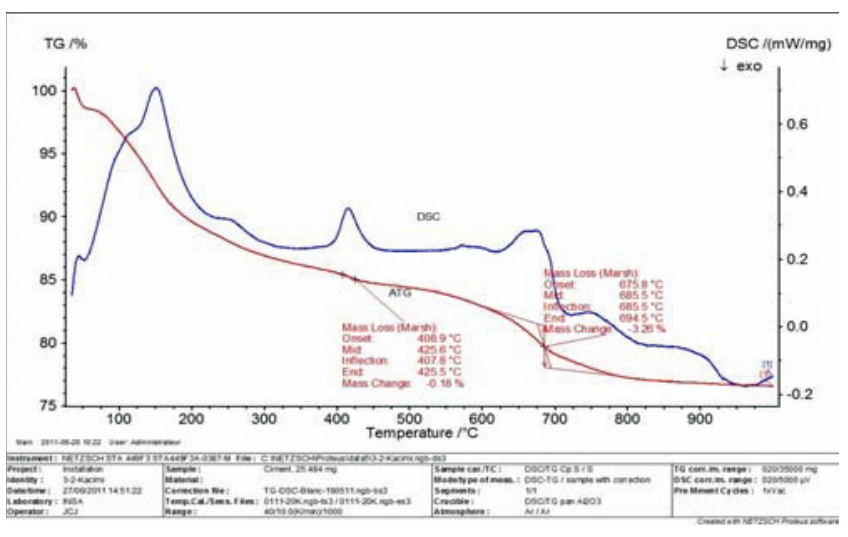

Fig. 4. Diagramme DSC-ATG de la pâte du mélange ChauxZéolithe X synthétisée

\subsection{Etude de la pouzzolanicité de la zéolithe synthétisée par DRX}

L'analyse par diffraction des rayons $\mathrm{X}$ de la pâte du mélange (Chaux hydraulique-Zéolithe $\mathrm{X}$ synthétisée), déjà préparée pour l'analyse DSC-ATG, a été utilisée pour confirmer l'activité pouzzolanique de cette zéolithe $\mathrm{X}$, après 2, 7 et 28 jours de durcissement. Les résultats obtenus sont donnés par le diffractogramme RX dans la figure 5 .

Les intensités des pics caractéristiques de la zéolithe $\mathrm{X}$ ont diminué avec une bonne formation d'une bosse entre 25 et $45^{\circ}$ qui est le domaine du gel de C-S-H/C-A$\mathrm{H}$. Les intensités des pics de portlandite diminuent avec l'âge de durcissement (de 2 à 28 jours), accompagnée d'une augmentaion de la bosse caractrésitique des C-S$\mathrm{H} / \mathrm{C}-\mathrm{A}-\mathrm{H}$. Ceci confirme que la portalndite a été consommée par la zéolithe pour former le gel par une réaction pouzzolanique.

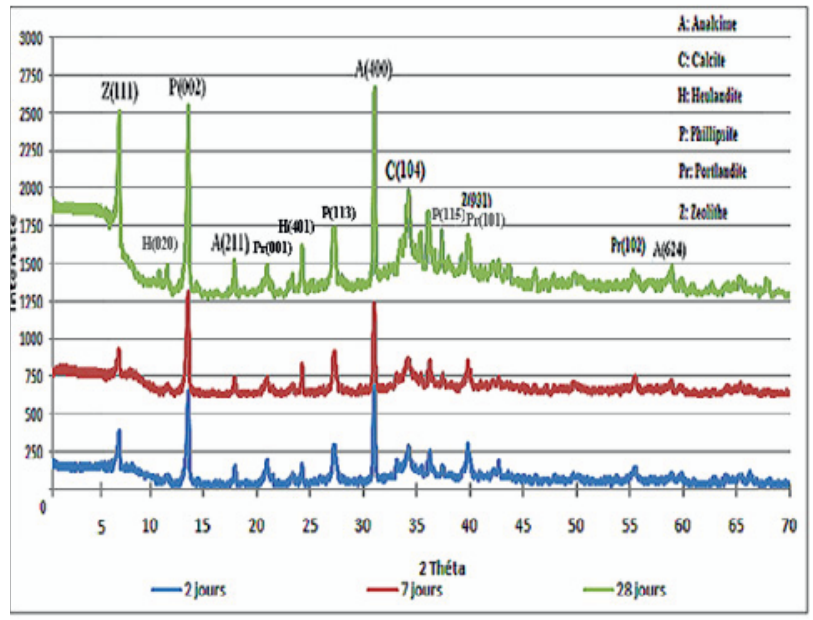

Fig. 5. Diffractogramme RX de la pâte du mélange ChauxZéolithe X synthétisée

\subsection{Temps de prise du ciment composé de zéolithe synthétisée}

Pour confirmer l'activité pouzzolanique de la zéolithe synthétisée, la réactivité hydraulique du ciment composé de 10 et $20 \%$ de cette zéolithe a été étudiée en la 
comparant au ciment témoin (sans addition) qui est de type CEM I 42,5 N. Un mini-moule tronconique de l'appareil de Vicat a été utilisé pour déterminer la prise des pâtes de ces ciments élaborées avec un rapport Eau/Ciment de 0,25. Des courbes de la variation de la hauteur de non pénétration de l'aiguille de Vicat avec le temps d'hydratation ont été tracées. Les résultats obtenus sont illustrés dans la figure 6 .

Les deux ciments élaborés ont une prise très rapide par rapport au témoin, donc ils ont une réactivité hydraulique importante dont leur durcissement est atteint dans un temps ne dépassant pas $50 \mathrm{mn}$ pour le ciment à $20 \%$ de zéolithe, et $150 \mathrm{mn}$ pour le ciment à $10 \%$ de zéolithe. Le ciment élaboré avec addition de $20 \%$ de zéolithe a une prise très rapide par rapport à celui de $10 \%$ de zéolithe, ce qui montre que la zéolithe $\mathrm{X}$ synthétisée a un effet important sur le temps de prise et donc sur l'hydratation. Ces caractéristiques confirment l'activité pouzzolanique de cette zéolithe montrée par les analyses de chaux saturée, thermique et de DRX.

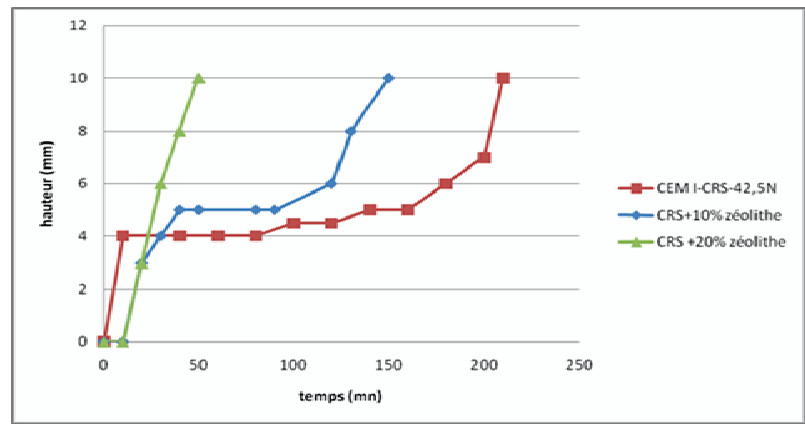

Fig. 6. Essai de prise sur des pâtes des ciments élaborés (ciment témoin et ciment à 10 et $20 \%$ de zéolithe X)

\subsection{Résistance mécanique des ciments élaborés}

La figure 7 représente la variation de la résistance à la compression des deux ciments èlaborès avec addition de 10 et $20 \%$ de zeolithe $X$ synthetisée et du ciment témoins après 2,7 et 28 jours de durcissement. Les résultats montrent que la résistance des deux ciments elaborés avec 10 et $20 \%$ de zeolithe est inferieure à celle du ciment temoin. La résistance à la compression du ciment élaboré avec $20 \%$ de zeolithe est plus grande par rapport à celle du ciment elaboré avec $10 \%$.

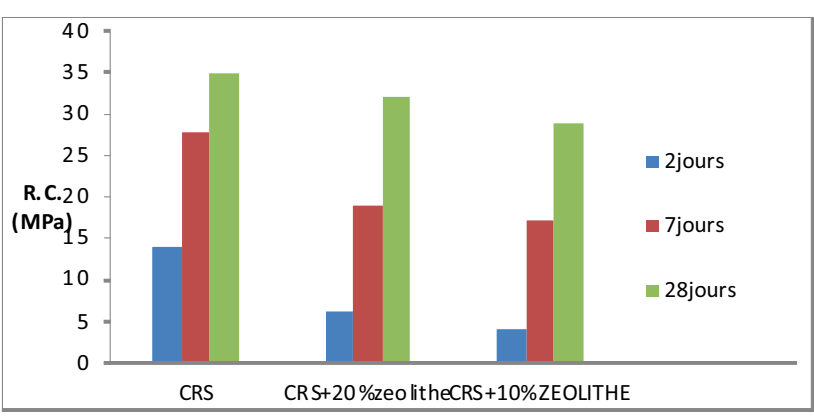

Fig 7. Résistances à la compression (R.C.) des ciments à 10 et $20 \%$ de zéolithe et le ciment témoin (CRS) après 2,7 et 28 jours de durcissement
La résistance à la compression des ciments composés de zéolithe $\mathrm{X}$ augmente rapidement avec le temps de 2 à 7 à 28 jours, cette évolution est plus rapide par comparaison au ciment témoin. L'addition de $20 \%$ de zéolithe améliore la résistance à la compression et son évolution dans le temps par rapport à l'addition de $10 \%$. Pour le ciment renfermant $20 \%$ de zéolithe la résistance mécanique à 28 jours est très proche de celle du ciment témoin. Ces résultats montrent que la zéolithe $\mathrm{X}$ synthétisée est pouzzolanique et à un effet positif sur le développement de la résistance mécanique, ce qui permet de substituer jusqu'à $20 \%$ de clinker par cette zéolithe active. L'addition de plus de $20 \%$ de cette zéolithe, voir 30 à $50 \%$, est recommandée pour des études ultérieures.

Ces résultats montrent que l'élaboration d'un écociment est possible par l'addition de la zéolithe $\mathrm{X}$, ce qui permet de réduire fortement les émissions de $\mathrm{CO}_{2}$ issus de la fabrication du clinker Portland.

\section{Conclusion}

L'élaboration d'un éco-ciment a été réalisée par la substitution partielle du clinker par la zéolithe $\mathrm{X}$ synthétisée à partir de kaolin naturel non riche en kaolinite. L'analyse par diffraction des rayons $\mathrm{X}$ a montré le caractère de cette zéolithe qui est de type $X$ avec une bonne cristallinité malgré la présence d'une phase amorphe. Cette phase amorphe n'est pas gênante dans cette étude car l'amorphe dans les aluminosilicates a aussi une activité pouzzolanique. Ces bonnes caractéristiques de cette zéolithe ont été obtenues malgré la nature de la matière utilisée dans la synthèse qui est un kaolin naturel algérien non pur.

L'activité pouzzolanique de la zéolithe $\mathrm{X}$ synthétisée a été étudiée par différentes techniques d'analyses et essais: test de chaux saturée, l'analyse thermique, la diffraction des rayons $\mathrm{X}$ et les essais physiques et mécaniques.

Les résultats du test de chaux saturée ont montré que cette zéolithe $\mathrm{X}$ synthétisée est fortement pouzzolanique dont le pourcentage de $\mathrm{CaO}$ consommée par la silice et l'alumine de la zéolithe dépasse $99 \%$ même à court terme ( 2 jours d'hydratation), et s'approche de $100 \%$ à 28 jours.

L'analyse par DSC-ATG confirme les résultats du test de chaux saturée par la bonne formation des gels C-S-H et $\mathrm{C}-\mathrm{A}-\mathrm{H}$ et la disparition quasi complète de la portlandite mélangée avec la zéolithe au départ. Ceci montre que la silice et l'alumine de la zéolithe sont fortement réactives et ont réagit rapidement avec la chaux hydraulique $\left[\mathrm{Ca}(\mathrm{OH})_{2}\right]$ par une réaction dite pouzzolanique, ce qui confirme alors le caractère pouzzolanique de cette zéolithe.

La diffraction des rayons $\mathrm{X}$ (DRX) montre que cette zéolithe est pouzzolanique ; une réduction des intensités des pics caractéristiques de la zéolithe $\mathrm{X}$ dans les pâtes du mélange (Chaux hydraulique-Zéolithe $\mathrm{X}$ ), a été constaté avec une bosse au milieu du diffractogramme qui témoigne la formation des C-S-H et C-A-H. Ces constatations indiquent que la réaction pouzzolanique entre la silice ou l'alumine de la zéolithe et la chaux additionnée a été réalisée, ce qui confirme les résultats 
obtenus par le test de chaux saturée et l'analyse thermique.

Pour confirmer ces résultats et montrer l'effet de l'addition la zéolithe $\mathrm{X}$ synthétisée sur les propriétés physiques et mécaniques du ciment composé, le temps de prise et la résistance à la compression des ciments composés de 10 et $20 \%$ de cette zéolithe ont été étudiés. Les résultats obtenus montrent que cette zéolithe améliore la réactivité hydraulique du ciment qui la renferme, ce qui permet d'augmenter la résistance mécanique de la pâte, malgré qu'elle reste légèrement inferieure par rapport à celle du ciment témoin (CEM I-CRS-42,5 N) après 28 jours de durcissement. L'évolution rapide de la résistance à la compression du ciment composé avec le temps et avec le pourcentage d'addition indique qu'une durée de durcissement prolongée ( 3 mois par exemple) et un taux de zéolithe $\mathrm{X}$ dépassant 20\% (30 à 50\%) permettront d'augmenter la résistance mécanique de ce ciment.

Ces résultats très satisfaisants permettent de conclure que la zéolithe $\mathrm{X}$ synthétisée est fortement pouzzolanique et peut être utilisée pour élaborer un éco-ciment tout en réduisant les émissions de $\mathrm{CO}_{2}$ résultant de la fabrication du clinker.

\section{Remerciement}

Les auteurs remercient vivement le Groupe Lafarge Algérie, le Centre National des Recherches Métallurgique CENIM-CSIC de Madrid, Espagne et le Laboratoire des Matériaux et Durabilité des Constructions- LMDCINSA-UPS-Toulouse, France, pour leurs soutiens techniques à la réalisation de ce travail.

\section{Références}

[1] D. Ravikumar, S. Peethamparan, N. Neithalath, Structure and strength of $\mathrm{NaOH}$ activated concretes containing fly ash or GGBFS as the sole binder», Cement and Concrete Composites 32 (2010) 399. 410.

[2] A. Sepulcre-Aguilar, F. Hernández-Olivares, Assessment of phase formation in lime-based mortars with added metakaolin, Portland cement and sepiolite, for grouting of historic masonry, Cement and Concrete Research 40 (2010) 66-76.

[3] S. Donatello, M. Tyrer, C. R. Cheeseman, Comparison of test methods to assess pozzolanic activity, Cement and Concrete Composites $\mathbf{3 2}$ (2010) 121-127.

[4] C. Defosse, Chimie du ciment, valorisation des déchets en cimenterie, Université libre de Bruwelles, site "CHIM 315 Partie III, France (2004).

[5] S. Chromy, High-temperature microphotometry and microdilatometry, Silikaty 18 (1974) 105-123. 